\title{
Reliable Clinical Serum Analysis with Reusable Electrochemical Sensor: Toward Point-of-Care Measurement of the Antipsychotic Medication Clozapine
}

\author{
Mijeong Kang ${ }^{\mathrm{a}, \mathrm{b}}$, Eunkyoung Kim ${ }^{\mathrm{a}, \mathrm{b}}$, Thomas E. Winkler ${ }^{\mathrm{b}, \mathrm{c}}$, George Banis ${ }^{\mathrm{b}, \mathrm{c}}$, Yi Liu ${ }^{\mathrm{a}, \mathrm{b}}$, \\ Christopher A. Kitchen ${ }^{d}$, Deanna L. Kelly ${ }^{d}$, Reza Ghodssis,c,e ${ }^{\text {, }}$, and Gregory F. Payne ${ }^{\text {a,b,* }}$ \\ ${ }^{a}$ Institute for Bioscience and Biotechnology Research and ${ }^{b}$ Fischell Department of \\ Bioengineering, University of Maryland, College Park, MD 20742, United States. \\ ${ }^{c}$ MEMS Sensors and Actuators Laboratory (MSAL), University of Maryland, College \\ Park, MD 20742, United States. \\ ${ }^{\mathrm{d}}$ Maryland Psychiatric Research Center, University of Maryland School of Medicine, \\ Baltimore, MD 21228, United States. \\ ${ }^{\mathrm{e}}$ Department of Electrical and Computer Engineering, Institute for Systems Research, \\ University of Maryland, College Park, MD 20742, United States. \\ * Corresponding author. Email: gpayne@umd.edu. Phone:1-301-405-8389; FAX: 1 - \\ 301-314-9075
}

\begin{abstract}
Clozapine is one of the most promising medications for managing schizophrenia but it is under-utilized because of the challenges of maintaining serum levels in a safe therapeutic range (1-3 $\mu \mathrm{M})$. Timely measurement of serum clozapine levels has been identified as a barrier to the broader use of clozapine, which is however challenging due to the complexity of serum samples. We demonstrate a robust and reusable electrochemical sensor with graphene-chitosan composite for rapidly measuring serum levels of clozapine. Our electrochemical measurements in clinical serum from clozapine-treated and clozapine-untreated schizophrenia groups are well correlated to centralized laboratory analysis for the readily detected uric acid and for the clozapine which is present at 100fold lower concentration. The benefits of our electrochemical measurement approach for serum clozapine monitoring are: (i) rapid measurement $(\approx 20 \mathrm{~min})$ without serum pretreatment; (ii) appropriate selectivity and sensitivity (limit of detection $0.7 \mu \mathrm{M}$ ); (iii)
\end{abstract}


reusability of an electrode over several weeks; and (iv) rapid reliability testing to detect common error-causing problems. This simple and rapid electrochemical approach for serum clozapine measurements should provide clinicians with the timely point-of-care information required to adjust dosages and personalize the management of schizophrenia.

Keywords: Therapeutic Drug Monitoring, Electrochemical Sensor, Serum, Clozapine, Graphene, Electrodeposition

\section{Introduction}

Schizophrenia is a devastating disorder that is poorly understood and difficult to manage. Clozapine (Wenthur and Lindsley, 2013) is one of the most promising medications for treating refractory schizophrenia and controlling violent behaviors and risks of suicide (Fakra and Azorin, 2012). Many experts believe that a broader use of clozapine would benefit both individuals and the larger society, but clinicians are often hesitant to prescribe clozapine because of its adverse side effects (Fakra and Azorin, 2012; Freudenreich et al., 2013; Gee et al., 2014; Kelly et al., 2007; Nielsen et al., 2012; Warnez and Alessi- Severini, 2014). In fact, clozapine's side effect risks led to its removal from the market, but it was later re-introduced because of its unique therapeutic benefits (Warnez and Alessi- Severini, 2014; Wenthur and Lindsley, 2013). As

illustrated in Figure 1a, the challenge to clinicians is that serum clozapine levels cannot be adequately controlled by dosage because of variabilities in drug metabolism (Couchman et al., 2013; Rajkumar et al., 2013; Rostami- Hodjegan et al., 2004). A recent survey indicated that clozapine would be more broadly prescribed if serum levels could be rapidly measured at the point-of-care (POC) to provide clinicians with the timely information required to adjust dosages (Kelly et al., 2015). 
(A) Clozapine level

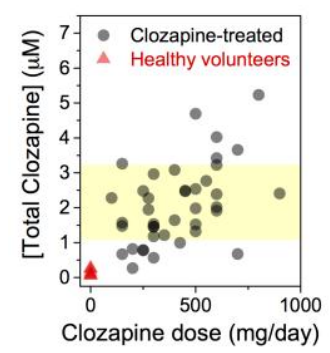

(B) Monitoring Clozapine in Serum

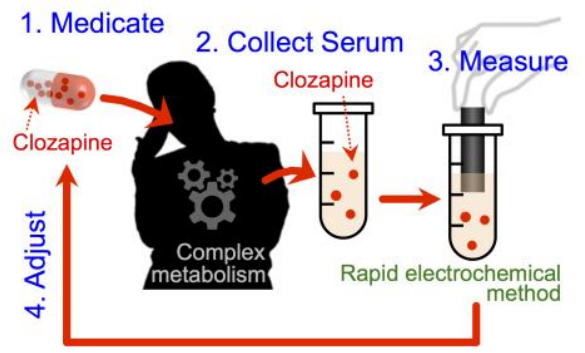

(C) Reliability Testing for Reuse

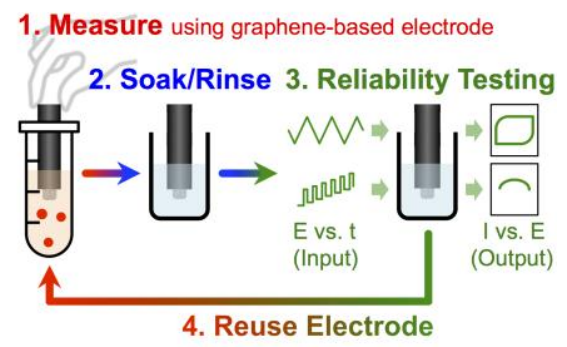

Figure 1. Rapid Therapeutic Drug Monitoring for the Antipsychotic Medication

Clozapine. (A) Clozapine is challenging to prescribe because it is active a low concentrations with narrow therapeutic window $(1-3 \mu \mathrm{M})$, and it is difficult to control serum levels by dosage because of significant variabilities in its metabolism. Total clozapine was measured from centralized laboratory and is the sum of clozapine and norclozapine. (B) Electrode measurements offer the potential for simple and rapid measurement of clozapine in human serum. (C) Experimental procedure for reusing a graphene composite-coated electrode.

Currently, clozapine is analyzed in centralized laboratories, using instrumentintensive methods (e.g., high-performance liquid chromatography and mass spectrometry) that require several days turn-around. Since clozapine has intrinsic electrochemical activity, a simple electrochemical method could possibly provide the timely POC analysis as illustrated in Figure 1b. In fact, several groups have begun developing electrochemical methods and significant progress has been made to achieve the high sensitivities required for clinical analysis in the relevant therapeutic range (1-3 $\mu \mathrm{M} ; 1000$ fold lower than serum glucose levels) (Table S1, Supporting Information). However, when these methods are extended to clozapine-spiked serum analysis, sensitivities typically decrease by orders-of-magnitude (increases in limit of detection). To date, we are unaware of any method that has succeeded in the clinic to provide the timely information needed to adjust clozapine dosages and personalize the management of schizophrenia.

Here, we report serum clozapine analysis with an electrode coated with an electrodeposited graphene-chitosan composite coating. Because of our translational aim, we focus on clinical serum analyses (Chan et al., 2016) from three groups, healthy 
controls, and schizophrenia groups with and without clozapine-treatment. Traditional thinking would suggest that the problems in extending analysis from buffer to serum are that serum contains components that foul the electrode or interfere with the analyte's signal (Barfidokht and Gooding, 2014; Capaldo et al., 2016; Coldur and Andac, 2013; Downard and bin Mohamed, 1999; Geise et al., 1991). Our evidence indicates that neither is the problem. Rather, we believe the problem in extending analysis from buffer to serum is the large and possibly variable binding of clozapine to serum proteins $(90-95 \%$ of clozapine is bound to serum proteins (Espnes et al., 2012; Flanagan et al., 2003; Leung et al., 2014; Schaber et al., 1998; Wu et al., 2011). Presumably, the unbound clozapine is measured electrochemically which may explain the diminished sensitivities when buffered solutions are compared against clozapine-spiked serum samples (E. Kim et al., 2015). It has also been suggested that only the unbound form of clozapine is biologically active (Lee et al., 2016), yet standard centralized laboratory analyses measure the total clozapine (protein bound plus unbound). While we acknowledge the limitations of this standard analytical method, these measurements are currently used by clinicians to adjust dosages and thus we use this standard to develop our electrochemical method.

The goal of this work is to develop a simple, rapid and reliable method for serum clozapine measurement within the constraint that we are comparing against a standard method that is not entirely comparable (standard method measures bound and unbound forms of clozapine). We demonstrate the validity of our electrochemical measurements using uric acid as a proxy, and show a high correlation between electrochemical measurements and standard centralized laboratory analysis of uric acid $(r=0.960, p<$ 0.001). As illustrated in Figure 1c, we devised simple, automatable electrochemical reliability tests to ensure reliable measurements over extended electrode use. We specifically report the following advantages for this electrochemical serum clozapine measurement: (i) serum pre-treatment and dilution are not required; (ii) selectivity and sensitivity (limit of detection $0.7 \mu \mathrm{M}$ ) are appropriate; (iii) measurements are rapid (20 minutes); (iv) the graphene-chitosan coating is stable allowing repeated use over several weeks; and (v) rapid, automatable electrochemical reliability tests can be used to detect common error-causing problems. 


\section{Materials and methods}

\subsection{Chemicals}

Chitosan, clozapine, norclozapine, and uric acid were purchased from SigmaAldrich. Graphene (N002-PDR) was purchased from Angstron Materials. Deionized water (>18 M $\Omega$ ) was obtained from Super-Q water system (Millipore). Chitosan solution (1.1\%; $\mathrm{pH} 5.3)$ was prepared by dissolving chitosan flakes in deionized water and the $\mathrm{pH}$ was adjusted using an $\mathrm{HCl}$ solution. A stock solution of clozapine $(5 \mathrm{mM})$ was prepared in methanol and stored at $-80{ }^{\circ} \mathrm{C}$. Standard clozapine solutions were prepared by diluting this stock solution with serum of healthy volunteers.

\subsection{Fabrication of Graphene-Chitosan-Coated Electrode}

Graphene-chitosan composite is deposited on a gold electrode, relying on the $\mathrm{pH}-$ responsive film-formation of chitosan (see Supporting Information for further details of the electrodeposition of the graphene-chitosan film) (Wan et al., 2011; Yang et al., 2013).

\subsection{Instrumentation}

To electrodeposit graphene-chitosan film, a DC power supply (2400 Sourcemeter, Keithley) was used. To perform electrochemical analyses, an electrochemical analyzer (CHI420a, CH Instruments) was used with a three-electrode configuration ( $\mathrm{Ag} / \mathrm{AgCl}$ (3 $\mathrm{M} \mathrm{KCl)} \mathrm{reference} \mathrm{electrode;} \mathrm{Pt} \mathrm{wire} \mathrm{counter} \mathrm{electrode).} \mathrm{Differential} \mathrm{pulse} \mathrm{voltammetry}$ was performed by scanning potential from 0 to $0.45 \mathrm{~V}$ at a scan rate of $2 \mathrm{mV} / \mathrm{s}(1 \mathrm{mV}$ step increment; every $0.5 \mathrm{~s})$ with superimposed pulses $(50 \mathrm{mV}$ pulse amplitude; $0.2 \mathrm{~s}$ pulse period). Cyclic voltammetry was performed by scanning potential from 0 to $0.6 \mathrm{~V}$ at a scan rate of $50 \mathrm{mV} / \mathrm{s}$.

\subsection{Clozapine Analysis in Serum}

Blood samples from 3 populations were collected at the Maryland Psychiatric Research Center, University of Maryland School of Medicine, between May, 2015 and August, 2016 and serum samples were collected from the supernatant of the centrifuged blood of each participant and stored frozen $\left(-80^{\circ} \mathrm{C}\right)$ before assay. Those sampled populations include 1) individuals with a DSM-IV diagnosis of Schizophrenia or 
Schizoaffective Disorder, who are taking clozapine, 2) individuals with either diagnosis, but not taking clozapine and 3) a control group consisting of individuals with neither diagnosis nor taking clozapine. All participants completed data collection procedures on a single appointment lasting 1-2 hrs, and involved up to $45 \mathrm{~mL}$ of blood collection for commercial and electrochemical detection of clozapine and uric acid. Blood chemistries were conducted at commercial laboratory (LabCorp) and were considered as a comparison methodology for our serum analysis. Further information on clinical research ethics is provided in Supporting Information.

\section{Results and discussion}

\subsection{Initial Electrochemical Characterization}

Initial characterization of electrochemical sensing performance was conducted using phosphate buffered (100 mM; pH 7.4) solutions of clozapine $(2 \mu \mathrm{M})$ and uric acid $(100 \mu \mathrm{M})$. A previous study identified uric acid as a major interfering species for clozapine analysis in serum because the potential regions for clozapine and uric acid oxidation overlap ( 0.3-0.4 V vs Ag/AgCl, measured by a glassy carbon electrode) (Chocron et al., 2015). Control measurements with a bare gold electrode and a chitosancoated gold electrode showed such overlap in clozapine and uric acid oxidation (see Figure S4 in Supporting Information).

Figure 2a illustrates the graphene-chitosan-coated electrode used in this study. For measurements, the graphene-chitosan-coated electrode was placed in the solution for $15 \mathrm{~min}$ (to allow diffusion into the graphene-chitosan composite film) and the analysis was performed using differential pulse voltammetry (DPV). Figure $\mathbf{2 b}$ shows that the DPV for the buffer is featureless, while the DPVs for clozapine and uric acid solutions have distinct current peaks at 0.34 and $0.17 \mathrm{~V}$, respectively. The observation that graphene lowers the overpotential for uric acid oxidation is consistent with previous studies that suggest tight $\pi-\pi$ staking interactions between graphene and uric acid facilitate electron transfer (further details are provided in Supporting Information) (Han et al., 2010; Pandikumar et al., 2014). These results indicate that graphene's electrocatalytic activities may allow peak separation between clozapine and uric acid

oxidation. [Note: Signals of other common endogenous interfering species, ascorbic acid 
(at $0.07 \mathrm{~V}$ ) and dopamine (no peak in $0-0.45 \mathrm{~V}$ ), are further separated from clozapine signal, Figure S5 in Supporting Information.]

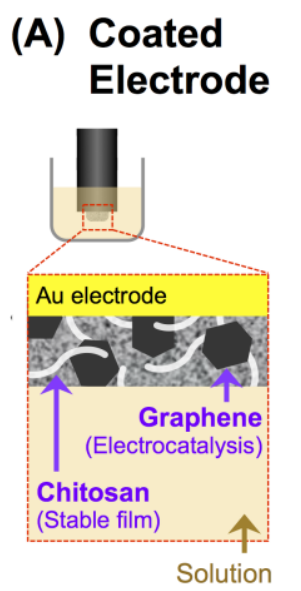

(B) Spiked Buffer Solution

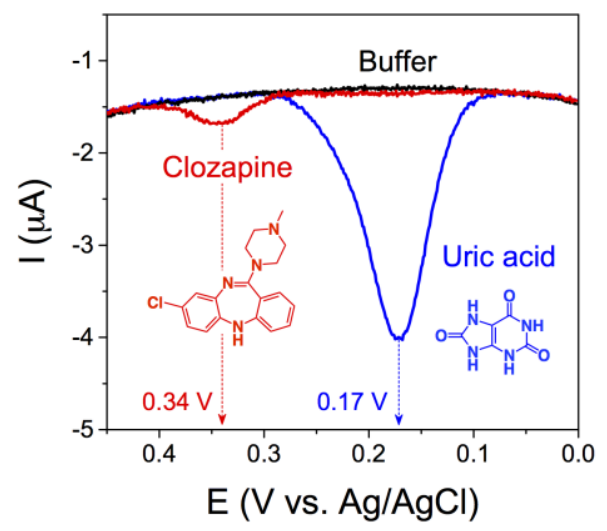

(D) Voltammetric Charge

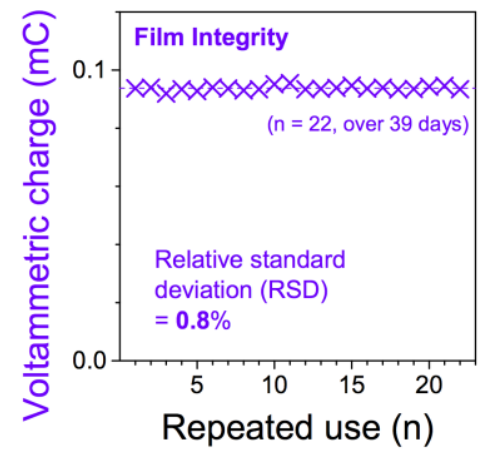

(E) Putative Uric Acid

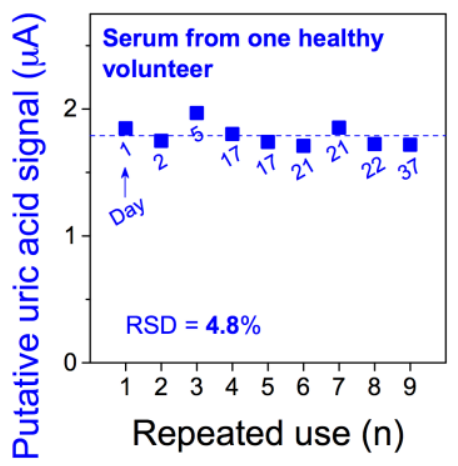

(C) Clinical Serum

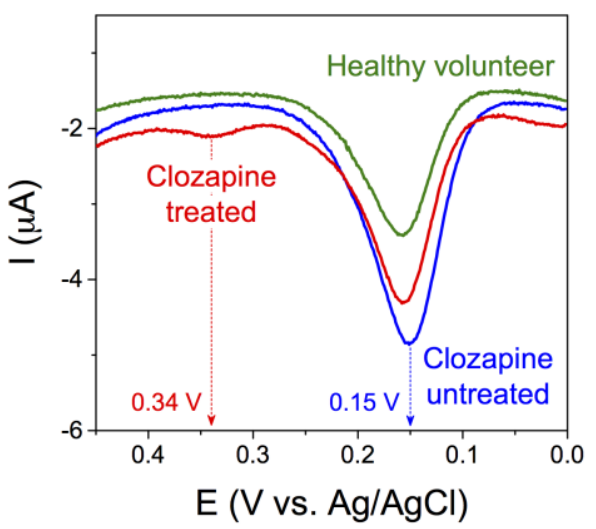

Figure 2. Characterization and Reliability of Graphene-Chitosan-Coated

Electrodes. (A) Schematic of a graphene-chitosan-coated electrode. (B) Differential pulse voltammograms (DPV) measured in phosphate buffered (100 mM; pH 7.4)

solutions of clozapine $(2 \mu \mathrm{M})$ or uric acid $(100 \mu \mathrm{M})$. (C) DPV measured in serum from a healthy volunteer and persons diagnosed with schizophrenia with or without clozapine treatment. (D) The integrity of the film-coated electrode was functionally evaluated by voltammetric charge measurements in a phosphate buffer (100 mM; pH 7.4). (E) Measurement of the putative uric acid signals from serum from one healthy volunteer (dashed line corresponds to the average of nine measurements and the numbers refer to the day of measurement). (F) Measurement of the clozapine signals from $2 \mu \mathrm{M}$ clozapine-spiked serum (dashed line corresponds to the average of nine measurements). 
We also used DPV to analyze norclozapine, the principal plasma metabolite of clozapine (Li et al., 2005). Norclozapine has biological activity (Li et al., 2005; Olianas et al., 2009; Weiner et al., 2004) and clinicians sometimes measure the sum of clozapine and norclozapine as a "total clozapine" level (total clozapine level is shown in Figure 1a) (Diaz et al., 2014). We observed no significant differences in the DPVs between clozapine and norclozapine (Figure S6, Supporting Information). Thus, our electrochemical signal at $0.34 \mathrm{~V}$ is a "total" clozapine (i.e., sum of clozapine and norclozapine) measurement (for simplicity, we will refer to this as clozapine signal).

We confirmed these initial DPV results using serum samples from a healthy volunteer and two people diagnosed with schizophrenia: one being treated with clozapine and the other not being treated with clozapine. As described above, a graphene-chitosancoated electrode was placed in the serum for $15 \mathrm{~min}$ before DPV measurement. Figure $2 \mathbf{c}$ shows that the DPV serum measurements from the healthy volunteer and clozapineuntreated person both show a large current peak at $0.15 \mathrm{~V}$ which is the same region as that for uric acid in Figure 2b. More importantly, all serum samples from 6 healthy volunteers and 3 clozapine-untreated persons show no peaks in the potential region for clozapine $(0.34 \mathrm{~V})$. This result suggests that serum components do not interfere with clozapine detection. Figure $2 \mathrm{c}$ shows that the serum from the clozapine-treated person has two DPV current peaks: a large peak in the region of uric acid and a smaller peak in the region of clozapine. These initial analyses suggest that the graphene-chitosan-coated electrode may offer sufficient sensitivity and selectivity for clozapine detection from human serum.

\subsection{Reuse and Reliability Testing}

We envision that a reusable sensor would provide greater reliability by avoiding the intrinsic variabilities that arise during sensor fabrication (Lin et al., 2009; Lubin et al., 2006; Ta et al., 2014). Electrode-to-electrode variability remains an unsolved practical challenge for electrochemical analysis (Lubin et al., 2006; Mahshid et al., 2015; Zhou and Rusling, 2001). To reuse our film-coated electrodes, we developed between-sample washing treatments and reliability tests to detect possible sources of errors. Figure S7 in Supporting Information shows the sequence of washing treatments and a rapid reliability 
test (DPV measurement in a buffer solution) developed to detect residual components that could contribute to false positives.

One of the major practical concerns for reusing a film-coated electrode is that the film could be damaged (e.g., scratched) during repeated use, and such damage could compromise subsequent measurements leading to an under-reporting of serum concentrations or even to false negatives. To detect such damage, we determined the film's charging current, which is associated with the area of electrically active surface and thus could indicate film integrity. We prepared a single graphene-chitosan-coated electrode and used it to perform multiple serum measurements over several weeks.

Figure 2d shows the voltammetric charge measurements (Chen et al., 2010) performed in a phosphate buffer solution before each use. The consistency of this reliability measure indicates that there is no observable deterioration in activity of the composite film (voltammetric charge $=0.0938 \mathrm{mC}$, relative standard deviation $(\mathrm{RSD})$ of $0.8 \%$ ) during the multiple measurements $(n=22$, over 39 days). Using the same electrode, we made 9 measurements of the putative uric acid signal in serum from a single healthy volunteer. [Note: Figure S8 in Supporting Information shows the method for quantifying the putative uric acid and clozapine signals.] Figure 2e shows this uric acid signal is stable (RSD 4.8\%). Importantly, as indicated in Figure 2e these repeated measurements were performed over the course of 37 days and the stability of this uric acid signal further demonstrates the robustness of the graphene-chitosan-coated electrode. Figure $2 \mathbf{f}$ shows 9 repeated clozapine measurements for spiked serum from several healthy volunteers, and these measurements remain stable with no obvious signal decay over time. The RSD for the 9 clozapine measurements with this single electrode is $5.9 \%$.

In summary, the results in Figure 2d-f indicate that the graphene-chitosan film is stable to repeated washing and the graphene-chitosan-coated electrodes can be used to perform multiple serum measurements. Importantly, rapid electrochemical reliability tests (i.e., voltammetric charge and DPV) can be used to inspect the film to ensure measurement reliability.

\subsection{Electrode Reuse Avoiding Electrode-to-Electrode Variability}


The reusability of a graphene-chitosan-coated electrode not only enabled repeated use of a single electrode but also avoided electrode-to-electrode variability. To illustrate this point, we analyzed a serum sample from a clozapine-treated individual using three separate composite coated electrodes. When the three serum clozapine signals from three electrodes are simply averaged, a high variability is observed (RSD 33.9\%, Figure S9a in Supporting Information). In contrast, if electrode-specific standard curves obtained from clozapine-spiked serum are used to covert the signal to the concentration, much lower variability is obtained in the calculated clozapine concentrations (RSD 9.4\%, Figure S9b and Figure S9c in Supporting Information). This experiment shows that the reusability of a graphene-chitosan-coated electrode and the electrode-specific standard curves enable more reproducible measurements.

\subsection{Serum Analysis of Schizophrenia Groups and Healthy Controls}

Finally, we evaluated the clinical feasibility (J. Kim et al., 2016) of our graphenechitosan-coated electrodes to analyze serum samples from a clozapine-treated schizophrenia group $(n=9)$. These serum samples were directly measured without sample pre-treatment (e.g., no deproteination or dilution). Each sample was measured in triplicate with the three separate electrodes and each electrode was reused with the washing procedure and reliability tests as shown in Figure S7 in Supporting Information. These electrochemical results were compared with independent measurements by a centralized laboratory (LabCorp) using standard methods for clozapine and uric acid detection. To further check the reliability of our measurements, additional comparisons were made with serum samples from 3 clozapine-untreated people with schizophrenia and 6 healthy volunteers.

The measured DPV signal at $\sim 0.15 \mathrm{~V}$ (putative uric acid signal) was simply averaged for the three electrodes. Figure 3a shows a high correlation between the DPV signals and the uric acid levels measured by the centralized laboratory (correlation coefficient, $\mathrm{r}=0.960, \mathrm{p}<0.001)$. This correlation supports our tentative assignment of the DPV signal at $0.15 \mathrm{~V}$ to uric acid. Further, this correlation indicates that our method can reliably extract quantitative information for metabolites that are present at high concentrations and that yield a robust signal. 


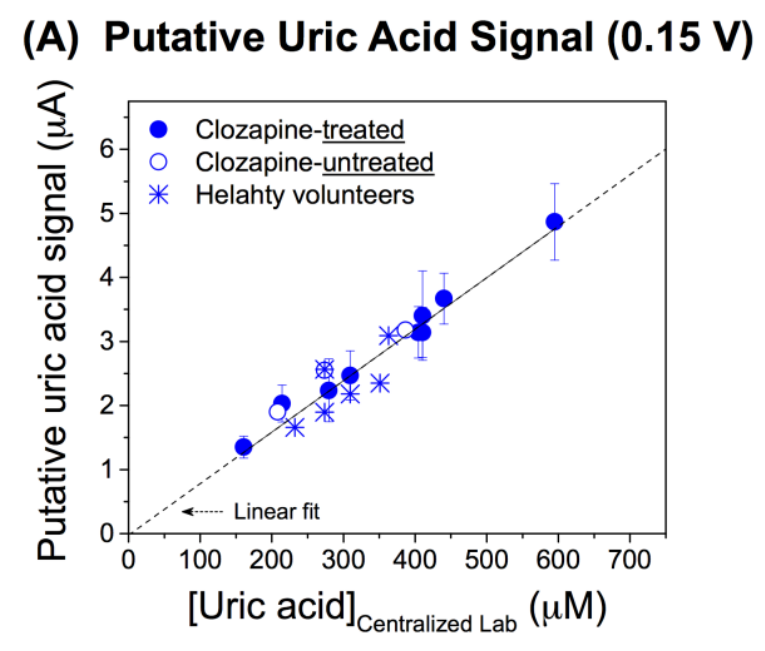

\section{(B) Clozapine Signal (0.34 V)}

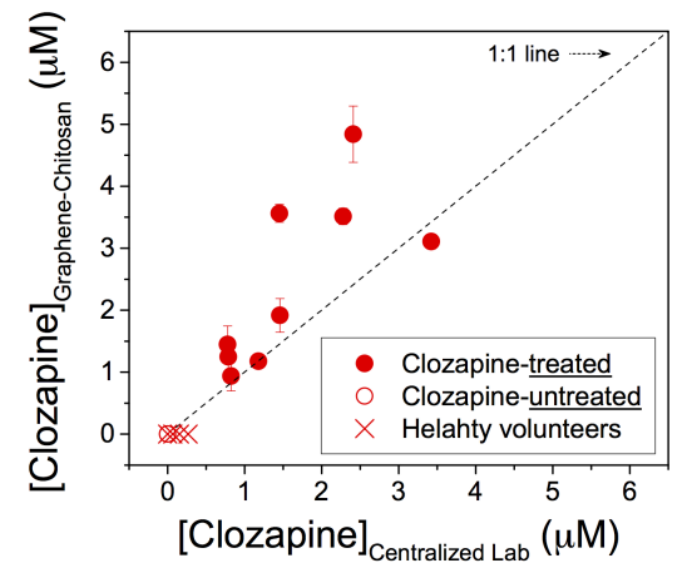

Figure 3. Correlation of Measurements between Graphene-Chitosan-Coated

Electrode and Centralized Laboratory. (A) Putative uric acid and (B) total clozapine signals from serum from healthy volunteers and persons with schizophrenia (with or without clozapine treatment). Measurements by graphene-chitosan-coated electrodes were obtained from three separate electrodes.

For clozapine analysis, the DPV signal ( 0.34 V) for each electrode was converted to a concentration using the electrode-specific calibration curves in Figure S9b obtained from clozapine-spiked serum (averged measurements indicate a limit of detection of $0.7 \mu \mathrm{M}$ as described in Supporting Information). Figure $\mathbf{3 b}$ shows the correlation between total clozapine concentrations measured by graphene-chitosan-coated electrodes and the centralized laboratory. The correlation indicates a strong association 
between the measurements $(r=0.729, p=0.026)$. At this point it is impossible to discern if the deviations reflect limitations in the electrochemical method or the standard method, or if they reflect the intrinsic differences in the two methods (e.g., standard method measures the sum of unbound and protein bound clozapine while electrochemistry likely measures unbound clozapine) and variabilities in the samples (e.g., differences in proteinbound clozapine between individuals). One noticeable feature in Figure $3 \mathrm{~b}$ is that no clozapine signals were observed in the DPV serum measurements from the clozapineuntreated people with schizophrenia and the healthy volunteers (i.e., no false positives were observed as shown in Figure S10 in Supporting Information). Thus, the graphenechitosan-coated electrode provides reliable measurements of serum clozapine levels.

\section{Conclusions}

Electrochemistry offers unique opportunities for rapid, sensitive and convenient POC analysis but remains underutilized in clinical practice. The translation of an electrochemical POC method into clinical practice will require a high level of confidence in the trustworthiness of the electrochemical signal. We believe this confidence will only result from extensive comparison to currently-used clinical methods for serum analysis (e.g., by centralized laboratories). Here we report a reusable graphene-chitosan coated electrode for the analysis of clozapine levels from clinical serum samples. We compared electrochemical and centralized laboratory analysis and show; a high correlation for serum uric acid levels which are readily measured $(\mathrm{r}=0.960, \mathrm{p}<0.001)$; and a good correlation for clozapine analysis despite the intrinsic differences between these measurements that result from clozapine's high affinity for protein binding $(r=0.729, p$ $=0.026)$. Further, we devised simple, automatable reliability tests to detect error-causing problems. These results demonstrate that a graphene-chitosan electrode allows the rapid and reliable electrochemical analysis of serum clozapine that could transform the management of schizophrenia by providing the timely information needed to adjust dosages of this promising medication. 


\section{Acknowledgements}

We gratefully acknowledge financial support from the United States: National Science Foundation (CBET-1435957), Defense Threat Reduction Agency (HDTRA1-131-0037), and the National Institutes of Health (R01 MH105571-01).

\section{References}

Barfidokht, A., Gooding, J.J., 2014. Electroanalysis 26, 1182-1196.

Capaldo, P., Alfarano, S.R., Ianeselli, L., Zilio, S.D., Bosco, A., Parisse, P., Casalis, L., 2016. ACS Sens. 1, 1003-1010.

Chan, W.C.W., Udugama, B., Kadhiresan, P., Kim, J., Mubareka, S., Weiss, P.S., Parak, W.J., 2016. ACS Nano 10, 8139-8142.

Chen, W., Fan, Z., Gu, L., Bao, X., Wang, C., 2010. Chem. Commun. 46, 3905-3907.

Chocron, S.E., Weisberger, B.M., Ben- Yoav, H., Winkler, T.E., Kim, E., Kelly, D.L., Payne, G.F., Ghodssi, R., 2015. PLoS ONE 10, e0116310.

Coldur, F., Andac, M., 2013. Electroanalysis 25, 732-740.

Couchman, L., Bowskill, S.V.J., Handley, S., Patel, M.X., Flanagan, R.J., 2013. Early Interv. Psychiatry 7, 122-130.

Diaz, F.J., Eap, C.B., Ansermot, N., Crettol, S., Spina, E., de Leon, J., 2014. Pharmacopsychiatry 47, 89-96.

Downard, A.J., bin Mohamed, A., 1999. Electroanalysis 11, 418-423.

Espnes, K.A., Heimdal, K.O., Spigset, O., 2012. Ther. Drug Monit. 34, 489-492.

Fakra, E., Azorin, J.-M., 2012. Expert Opin. Pharmacother. 13, 1923-1935.

Flanagan, R.J., Yusufi, B., Barnes, T.R.E., 2003. Br. J. Clin. Pharmacol. 56, 135-138.

Freudenreich, O., Henderson, D.C., Sanders, K.M., Goff, D.C., 2013. Acad. Psychiatry $37,27-30$.

Gee, S., Vergunst, F., Howes, O., Taylor, D., 2014. Acta Psychiatr. Scand. 130, 16-24.

Geise, R.J., Adams, J.M., Barone, N.J., Yacynych, A.M., 1991. Biosens. Bioelectron. 6, 151-160.

Han, D., Han, T., Shan, C., Ivaska, A., Niu, L., 2010. Electroanalysis 22, 2001-2008.

Kelly, D.L., Ben- Yoav, H., Payne, G.F., Winkler, T.E., Chocron, S.E., Kim, E., Stock, V., Vyas, G., Love, R.C., Wehring, H.J., Sullivan, K.M., Feldman, S., Liu, F., McMahon, R.P., Ghodssi, R., 2015. Clin. Schizophr. Relat. Psychoses Epub ahead of print. 
Kelly, D.L., Kreyenbuhl, J., Dixon, L., Love, R.C., Medoff, D., Conley, R.R., 2007. Schizophr. Bull. 33, 1221-1224.

Kim, E., Chocron, S.E., Ben- Yoav, H., Winkler, T.E., Liu, Y., Glassman, M., Wolfram, C., Kelly, D.L., Ghodssi, R., Payne, G.F., 2015. Adv. Funct. Mater. 25, 2156-2165.

Kim, J., Biondi, M.J., Feld, J.J., Chan, W.C.W., 2016. ACS Nano 10, 4742-4753.

Lee, L.H.N., White, R.F., Barr, A.M., Honer, W.G., Procyshyn, R.M., 2016. J. Psychiatry Neurosci. 41, E67-E68.

Leung, J.G., Nelson, S., Takala, C.R., Goren, J.L., 2014. Ann. Pharmacother. 48, 801805.

Li, Z., Huang, M., Ichikawa, J., Dai, J., Meltzer, H.Y., 2005. Neuropsychopharmacology 30, 1986-1995.

Lin, S.P., Pan, C.Y., Tseng, K.C., Lin, M.C., Chen, C.D., Tsai, C.C., Yu, S.H., Sun, Y.C., Lin, T.W., Chen, Y.T., 2009. Nano Today 4, 235-243.

Lubin, A.A., Lai, R.Y., Baker, B.R., Heeger, A.J., Plaxco, K.W., 2006. Anal. Chem. 78, 5671-5677.

Mahshid, S.S., Camiré, S., Ricci, F., Vallée- Bélisle, A., 2015. J. Am. Chem. Soc. 137, 15596-15599.

Nielsen, J., Røge, R., Schjerning, O., Sørensen, H.J., Taylor, D., 2012. Eur. Neuropsychopharmacol. 22, 818-824.

Olianas, M.C., Dedoni, S., Ambu, R., Onali, P., 2009. Eur. J. Pharmacol. 607, 96-101.

Pandikumar, A., How, G.T.S., See, T.P., Omar, F.S., Jayabal, S., Kamali, K.Z., Yusoff, N., Jamil, A., Ramaraj, R., John, S.A., Lim, H.N., Huang, N.M., 2014. RSC Adv. 4, 63296-63323.

Rajkumar, A.P., Poonkuzhali, B., Kuruvilla, A., Jacob, M., Jacob, K.S., 2013. Int. Clin. Psychopharmacol. 28, 50-56.

Rostami- Hodjegan, A., Amin, A.M., Spencer, E.P., Lennard, M.S., Tucker, G.T., Flanagan, R.J., 2004. J. Clin. Psychopharmacol. 24, 70-78.

Schaber, G., Stevens, I., Gaertner, H.J., Dietz, K., Breyer- Pfaff, U., 1998. Br. J. Clin. Pharmacol. 46, 453-459.

Ta, V.T., Park, J., Park, E.J., Hong, S., 2014. ACS Nano 8, 2206-2213.

Wan, Y., Lin, Z., Zhang, D., Wang, Y., Hou, B., 2011. Biosens. Bioelectron. 26, 19591964.

Warnez, S., Alessi- Severini, S., 2014. BMC Psychiatry 14, 102.

Weiner, D.M., Meltzer, H.Y., Veinbergs, I., Donohue, E.M., Spalding, T.A., Smith, T.T., Mohell, N., Harvey, S.C., Lameh, J., Nash, N., Vanover, K.E., Olsson, R., Jayathilake, K., Lee, M., Levey, A.I., Hacksell, U., Burstein, E.S., Davis, R.E., Brann, M.R., 2004. Psychopharmacology 177, 207-216.

Wenthur, C.J., Lindsley, C.W., 2013. ACS Chem. Neurosci. 4, 1018-1025.

Wu, X., Liu, J., Wang, Q., Xue, W., Yao, X., Zhang, Y., Jin, J., 2011. Spectrochim. Acta, Part A 79, 1202-1209.

Yang, S., Lu, Z., Luo, S., Liu, C., Tang, Y., 2013. Microchim. Acta 180, 127-135.

Zhou, L., Rusling, J.F., 2001. Anal. Chem. 73, 4780-4786. 


\section{Graphical Abstract}

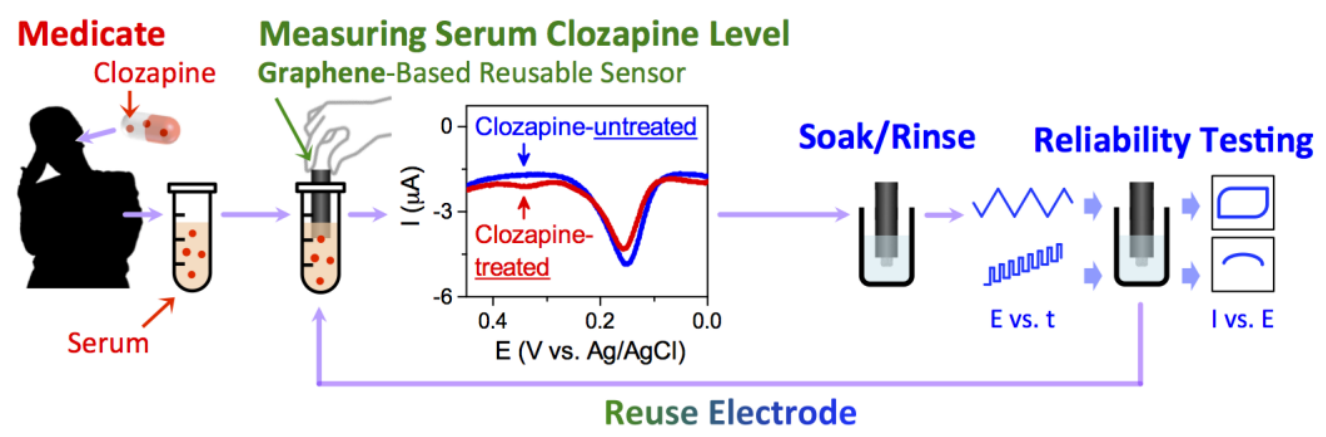

